\title{
OPEN Superior operational stability of immobilized L-asparaginase over surface-modified carbon nanotubes
}

Mafalda R. Almeida ${ }^{1,4}$, Raquel O. Cristóvão 2,4 , Maria A. Barros ${ }^{2}$, João C. F. Nunes ${ }^{1}$ Rui A. R. Boaventura' ${ }^{2}$, José M. Loureiro ${ }^{2}$, Joaquim L. Faria², Márcia C. Neves ${ }^{1}$, Mara G. Freire ${ }^{1}$, Valéria C. Santos-Ebinuma ${ }^{3}$, Ana P. M. Tavares ${ }^{1 \bowtie}$ \& Cláudia G. Silva ${ }^{2 \bowtie}$

L-asparaginase (ASNase, EC 3.5.1.1) is an enzyme that catalyzes the L-asparagine hydrolysis into L-aspartic acid and ammonia, being mainly applied in pharmaceutical and food industries. However, some disadvantages are associated with its free form, such as the ASNase short half-life, which may be overcome by enzyme immobilization. In this work, the immobilization of ASNase by adsorption over pristine and modified multi-walled carbon nanotubes (MWCNTs) was investigated, the latter corresponding to functionalized MWCNTs through a hydrothermal oxidation treatment. Different operating conditions, including $\mathrm{pH}$, contact time and ASNase/MWCNT mass ratio, as well as the operational stability of the immobilized ASNase, were evaluated. For comparison purposes, data regarding the ASNase immobilization with pristine MWCNT was detailed. The characterization of the ASNase-MWCNT bioconjugate was addressed using different techniques, namely Transmission Electron Microscopy (TEM), Thermogravimetric Analysis (TGA) and Raman spectroscopy. Functionalized MWCNTs showed promising results, with an immobilization yield and a relative recovered activity of commercial ASNase above $95 \%$ under the optimized adsorption conditions ( $\mathrm{pH}$ $8,60 \mathrm{~min}$ of contact and $1.5 \times 10^{-3} \mathrm{~g} \mathrm{~mL}^{-1}$ of ASNase). The ASNase-MWCNT bioconjugate also showed improved enzyme operational stability ( 6 consecutive reaction cycles without activity loss), paving the way for its use in industrial processes.

L-asparaginase (ASNase, EC 3.5.1.1) is an enzyme that catalyzes L-asparagine hydrolysis into L-aspartic acid and ammonia ${ }^{1}$, being present in different microorganisms such as bacteria, fungi, plant tissues, algae and in some animals ${ }^{2}$. This enzyme has important applications, mainly in the food and pharmaceutical industries. Specifically, in the food industry, ASNase prevents the formation of acrylamide (formed by the Maillard reaction that occurs at extreme temperatures between $\mathrm{L}$-asparagine and carbonyl compounds), a neurotoxic, genotoxic, carcinogenic, and toxic compound ${ }^{1}$. On the other hand, in the pharmaceutical industry, ASNase is used as a biopharmaceutical to treat acute lymphoblastic leukemia and other malignant diseases, including Hodgkin's disease and different types of leukemia or sarcoma ${ }^{3}$. The chemotherapeutic effect of ASNase is based on the catalysis of the L-asparagine present in blood, an essential amino acid for the growth of both normal and cancer cells ${ }^{4}$. Since cancer cells do not have L-asparagine synthetase, they must use the existing L-asparagine in the blood. Thus, ASNase achieves its antileukemic effect by depleting circulating asparagine and depriving cancer cells of asparagine ${ }^{3}$. Additionally, ASNase can be used in biosensor technology to detect asparagine levels in both sectors ${ }^{3}$. For instance, Li et al. ${ }^{5}$ produced an ammonium selective sensor based on adsorbed thermostable and recombinant ASNase biosensor from Archaeoglobus fulgidus cloned and expressed in Escherichia coli.

ASNase currently commercialized is produced from two primary bacterial sources, namely both recombinant E. coli and Erwinia chrysanthemi. Due to characteristics of its recombinant origin, its half-life and intrinsic

\footnotetext{
${ }^{1}$ CICECO-Aveiro Institute of Materials, Department of Chemistry, University of Aveiro, 3810-193 Aveiro, Portugal. ' Laboratory of Separation and Reaction Engineering - Laboratory of Catalysis and Materials (LSRE-LCM), Department of Chemical Engineering, Faculty of Engineering, University of Porto, Rua do Dr. Roberto Frias, 4200-465 Porto, Portugal. ${ }^{3}$ Department of Engineering Bioprocess and Biotechnology, School of Pharmaceutical Sciences, São Paulo State University (Unesp), Araraquara, Brazil. ${ }^{4}$ These authors contributed equally: Mafalda R. Almeida and Raquel O. Cristóvão. ${ }^{\circledR}$ email: aptavares@ua.pt; cgsilva@fe.up.pt
} 
activity decrease while its immunogenicity increases ${ }^{2}$. To overcome these disadvantages, Ulu and Ates ${ }^{3}$ recently reviewed natural and synthetic carriers (from erythrocytes to poly(ethylene glycol) (PEG)) and different techniques (chemical and physical methods) applied for ASNase immobilization. Immobilized enzymes have been widely used due to their improved stability and performance, and reusability ${ }^{6}$. However, the selection of the solid support and the procedure adopted for the enzyme immobilization significantly influences the resulting properties ${ }^{6}$.

Currently, nanomaterials and nanostructures have been widely applied as carriers for enzymes or proteins because of their high surface area and high enzyme/protein loading capacity ${ }^{7}$. Nanomaterials include several structures like nanoparticles, nanofibers and nanotubes, of which carbon nanotubes (CNTs) have been under extensive research in different fields, such as optics, electronics and catalysis ${ }^{8}$. CNTs are formed by a network of carbon atoms (graphene sheets) assembled into seamless cylinders of one (single-walled carbon nanotubes, SWCNTs, with a diameter of 0.4 to $3 \mathrm{~nm}$ ) ${ }^{9}$ or more layers (multi-walled carbon nanotubes, MWCNTs, with diameters of about 5 to $100 \mathrm{~nm}$ ) with either open or closed ends ${ }^{10}$. Additionally, MWCNTS can be classified according to two possible arrangements with implications on their properties: when the graphene sheets are arranged in nested concentric cylinders and when one graphene sheet is spirally wraparound on itself ${ }^{11}$. Moreover, CNTs' surfaces can be easily functionalized, tuning their properties towards specific applications and enhancing their efficiency either as supports or catalysts ${ }^{12-14}$.

CNTs have been reported as promising nanomaterials for diverse (bio)applications since relatively strong chemical interactions between biomolecules and CNTs are stablished, increasing the stability of the immobilized biomolecules towards harsh reaction conditions and enhance its reusability ${ }^{15}$. Specifically, the interaction of CNTs with biomolecules such as enzymes and proteins has been studied for laccase, peroxidase, organophosphate hydrolase, esterase, fibronectin, catalase, soy protein, among others ${ }^{12,13,16-25}$. The two main approaches for biomolecule attachment on CNTs are noncovalent and covalent immobilization. In the first one, the biomolecule is physically adsorbed on the CNT by hydrogen bonding or by hydrophobic, electrostatic, or $\pi-\pi$ stacking interactions. In the second one, the biomolecule is covalently linked to the CNT by esterification or amidation of previously oxidized CNTs, by covalent attachment of the functional groups over the CNTs surface, or by linking molecules, which act as "bridges" between the CNTs and the biomolecule ${ }^{26}$.

Regarding CNTs biocompatibility envisioning the aforementioned applications, recent studies have been reporting the reduction of CNTs toxicity through their functionalization. For instance, Dong et al. ${ }^{27}$ showed that acid oxidation of CNTs leads to a decrease of CNTs cytotoxicity; their biocompatibility was improved when under direct incubation with human epithelial cells (BEAS-2B cells) and with enzymes (soybean peroxidase). Jain et al. ${ }^{28}$ evaluated the in vivo toxicity of pristine and acid-oxidized multi-walled carbon nanotubes. It was found that acidic MWCNTs are less toxic and more biocompatible than their pristine counterparts, and that the toxicity of MWCNTs depends on their functionalization density, i.e., the higher the density of surface carboxyl groups, the lower was the MWCNTs toxicity ${ }^{28}$. Moreover, quantitative biodistribution studies in mice showed a rapid elimination of highly oxidized MWCNTs from the organism, while pristine and less oxidized MWCNTs revealed greater retention time ${ }^{28}$.

In a previous work we have found that ASNase may be efficiently immobilized by a simple physical adsorption method over pristine carbon nanotubes ${ }^{29}$. Although we have obtained very promising results in terms of ASNase activity, the presence of stronger interactions between the carbon phase and the enzyme is highly desired from the technological point of view since they normally result in bioconjugates with higher reusability ${ }^{29}$. Surface functionalization of CNTs is generally applied for this purpose.

To the best of our knowledge, up to now only two works have been published on the immobilization of ASNase over functionalized CNTs ${ }^{30,31}$. Ulu et al. ${ }^{30}$ reported the use of calcium-alginate/MWCNTs hybrid beads for the immobilization E. coli ASNase while Haroun et al. ${ }^{31}$ described the immobilization of partial purified Aspergillus versicolor ASNase onto oxidized MWCNTs.

In the present work, MWCNTs functionalized through a simple hydrothermal oxidation route with nitric acid were used for the immobilization of commercial E. coli ASNase and the results compared with those obtained with the unmodified material ${ }^{29}$, and by Haroun et al. $^{31}$, which used similar materials. Although, we propose a comparable strategy to the one developed applied by Haroun et $\mathrm{al}^{31}$, the immobilization process developed by us used an ASNase from a different microorganism (Haroun et al. ${ }^{31}$ immobilized ASNase produced by a fungi, A. versicolor, while we immobilized ASNase produced by a bacteria, E. coli). Although, both microorganisms are good sources of ASNase, the fungal complex morphology can be critical for the feasibility of scaling up the process since fungal cultivation in bioreactor is sensible to several parameters, such as oxygen supply and transfer, inoculum size, $\mathrm{pH}$, and stirring. Both enzymes have a base of similarity, but present significant differences in what concerns their structure and physicochemical properties ${ }^{1}$. In this sense, the immobilization behavior can be significantly different and for this reason we developed the present work to expand the characterization of $E$. coli ASNase immobilization. Different operation conditions, including pH, contact time and ASNase/ MWCNT mass ratio were evaluated. The immobilization of the enzyme was evidenced by characterization of the ASNase-MWCNT bioconjugate using different techniques. Moreover, the operational stability was assessed by determining the efficiency of the ASNase/MWCNT over a series of consecutive cycles.

\section{Results and discussion}

ASNase immobilization on functionalized MWCNTs. The effect of several parameters, namely $\mathrm{pH}$, contact time and ASNase/MWCNT mass ratio, on the enzyme immobilization yield and enzymatic activity was evaluated. The ASNase-MWCNTs bioconjugates were prepared using MWCNTs functionalized with different $\mathrm{HNO}_{3}$ concentrations, and for comparison purposes, pristine MWCNT was also tested. 
MWCNT-NC-0.05

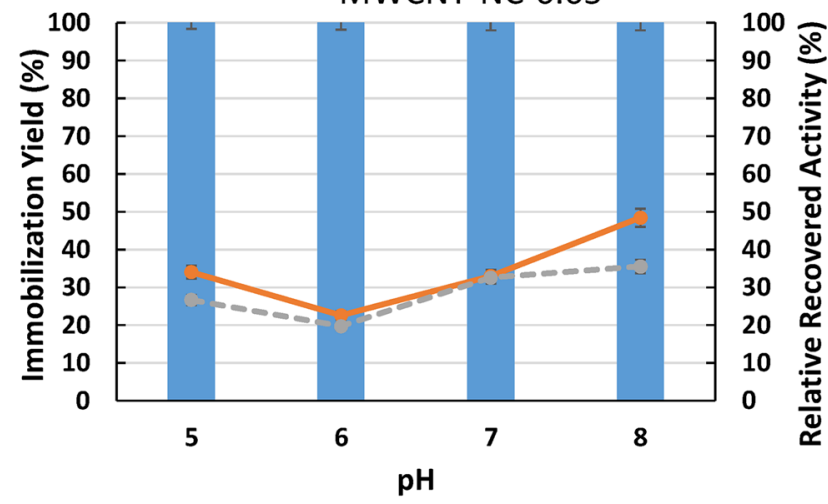

MWCNT-NC-0.2

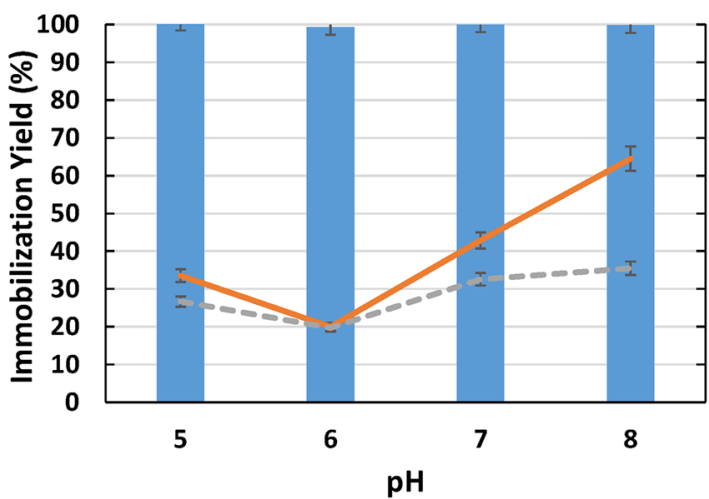

MWCNT-NC-0.1

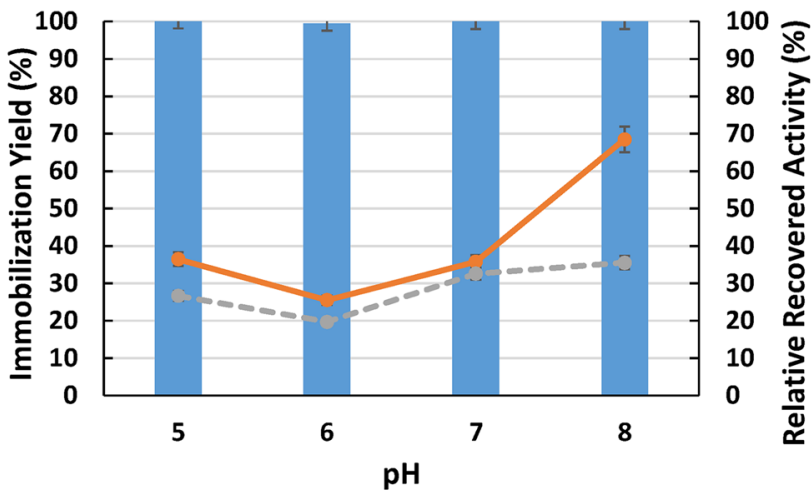

MWCNT-NC-0.3

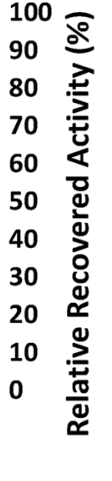

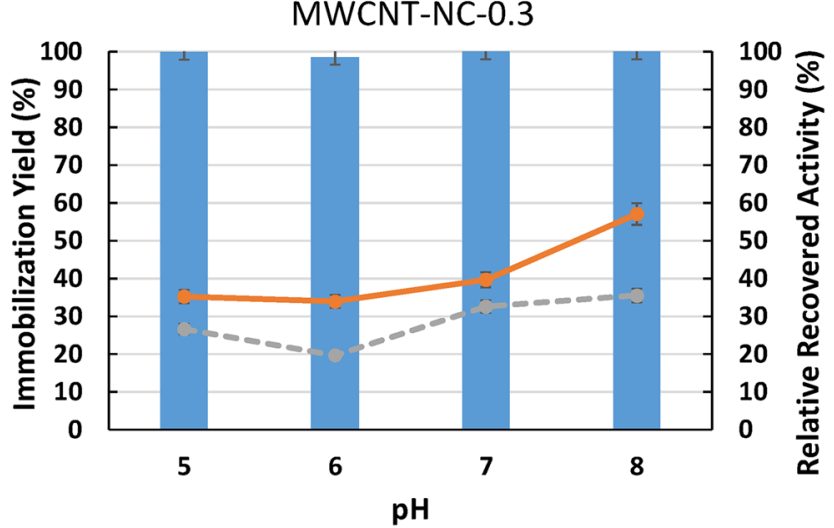

Figure 1. Effect of $\mathrm{pH}$ on immobilization yield (columns) and relative recovered activity (orange lines) obtained with the immobilization of $8.6 \times 10^{-5} \mathrm{~g} \mathrm{~mL}^{-1}$ of ASNase on $2 \mathrm{mg}$ of functionalized MWCNTs (functionalization with $\mathrm{HNO}_{3}$ aqueous solutions with variable concentrations: $0.05,0.10,0.20$ and $0.30 \mathrm{M}$ ) for $60 \mathrm{~min}$ of contact time. Grey lines represent the relative recovered activity of ASNase on $2 \mathrm{mg}$ of MWCNTs without any functionalization (MWCNT-NC, control).

Effect of the $p H$ on ASNase immobilization. In order to evaluate possible electrostatic interactions between ASNase and MWCNTs, firstly, the $\mathrm{pH}$ effect was evaluated at distinct $\mathrm{pH}$ values ranging from 5.0 to 8.0, whilst keeping the ASNase concentration at $8.6 \times 10^{-5} \mathrm{~g} \mathrm{~mL}^{-1}\left(1.72 \times 10^{-5} \mathrm{~g}\right.$ of ASNase) and contact time of $60 \mathrm{~min}$. To this end, the isoelectric point of ASNase was determined, obtaining a value of 5.2 (Figure S1 in the Supporting Information). This value follows the literature, where isoelectric points of ASNase ranging from 5.0 to 5.7 have been reported ${ }^{32}$. The results of ASNase immobilization yield and relative recovered activity are depicted in Fig. 1 (detailed data in Table S1 in the Supporting Information). According to Fig. 1, practically total enzyme adsorption was achieved in the $\mathrm{pH}$ range under study, with an immobilization yield above $98 \%$ (more than $1.62 \times 10^{-5} \mathrm{~g}$ of ASNase was immobilized) for all functionalized MWCNTs.

Under the studied conditions, the enzyme is partially positively charged in the assays performed at pH 5.0; at pH 6 and above, the enzyme is negatively charged. Additionally, as the point of zero charge of the modified MWCNTs used is around 3.0, the nanomaterials are negatively charged in all the assays performed ${ }^{22}$, which agrees with the metallic-like conductive properties of these materials. Thus, since in most $\mathrm{pH}$ values addressed the enzyme and MWCNTs are negatively charged, a repulsion between them would expected if electrostatic interactions are playing a role. Furthermore, no significant differences exist in the immobilization yields obtained at and above $\mathrm{pH}$. Therefore, electrostatic interactions between the material and the enzyme do not play a major role, with hydrophobic (hydrophobic regions on enzyme exterior can interact with the wall of CNTs through hydrophobic interaction) and $\pi-\pi$ stacking interactions (interaction between the sidewalls of CNTs and the ASNase aromatic rings) appearing as the major forces in the adsorption of ASNase ${ }^{33}$.

High ASNase relative recovered activities (RRA) were detected at $\mathrm{pH}$ 8.0, and low RRA at $\mathrm{pH}$ 6.0, meaning that the $\mathrm{pH}$ influences the activity of ASNase after immobilization. Probably, at low $\mathrm{pH}$ values (below 7.5), the active site of the immobilized enzyme is partially deactivated since it is known that ASNase is sensitive to high $\mathrm{H}^{+}$ concentrations ${ }^{34,35}$. Regarding the effect of the MWCNTs functionalization on the ASNase RRA, it is evident that a higher RRA was obtained when functionalized MWCNTs are used (Fig. 1-orange lines) over non-functionalized ones (Fig. 1-grey lines). Moreover, a similar tendency of the RRA increase with the increase in pH is observed for all degrees of MWCNTs surface functionalization. A maximum RRA of $69 \%$ was attained with the functionalized MWCNT-NC-0.1, followed by MWCNT-NC-0.2, MWCNT-NC-0.3 and MWCNT-NC-0.05. A detailed discussion about the effect of surface modification is presented below, together with the effect of contact time.

Considering high ASNase RRA values were detected at $\mathrm{pH}$ 8.0, this $\mathrm{pH}$ was selected for further assays. 
MWCNT-NC-0.05

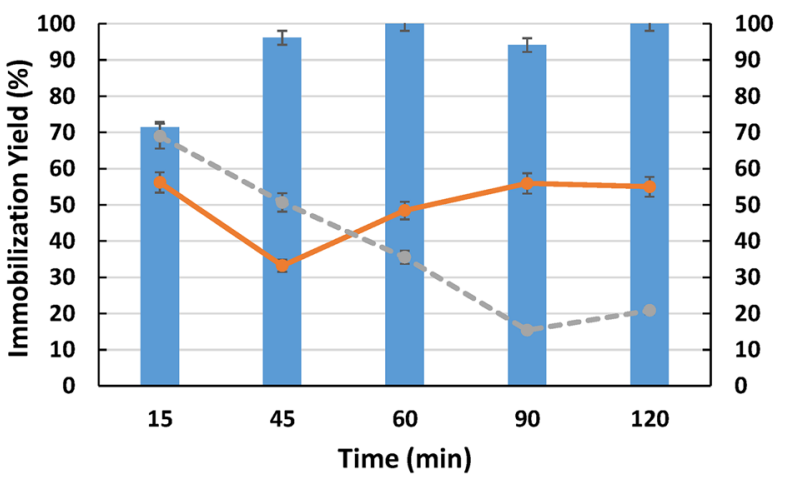

MWCNT-NC-0.2

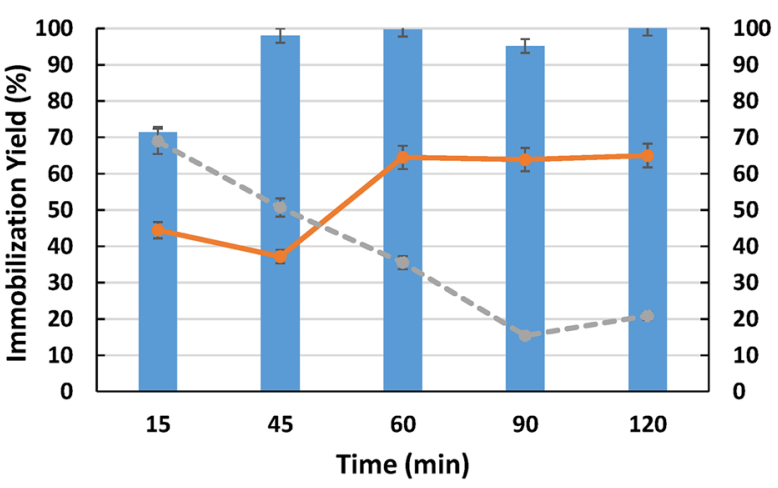

MWCNT-NC-0.1

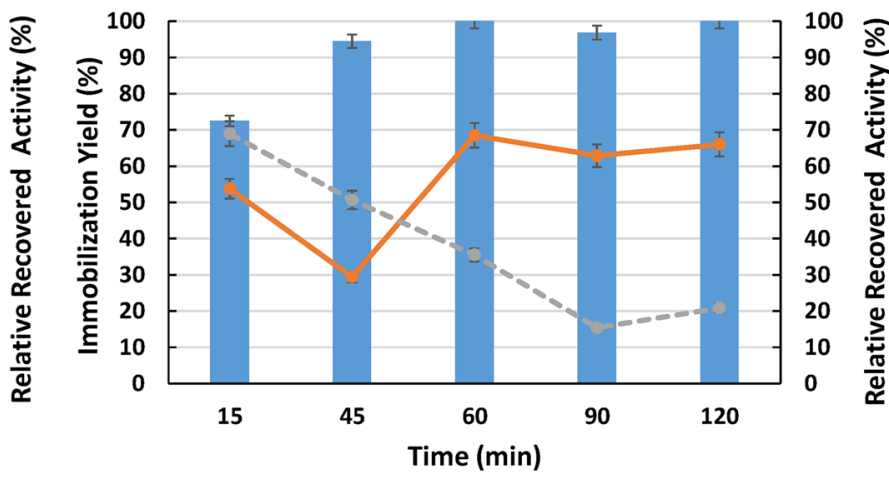

MWCNT-NC-0.3

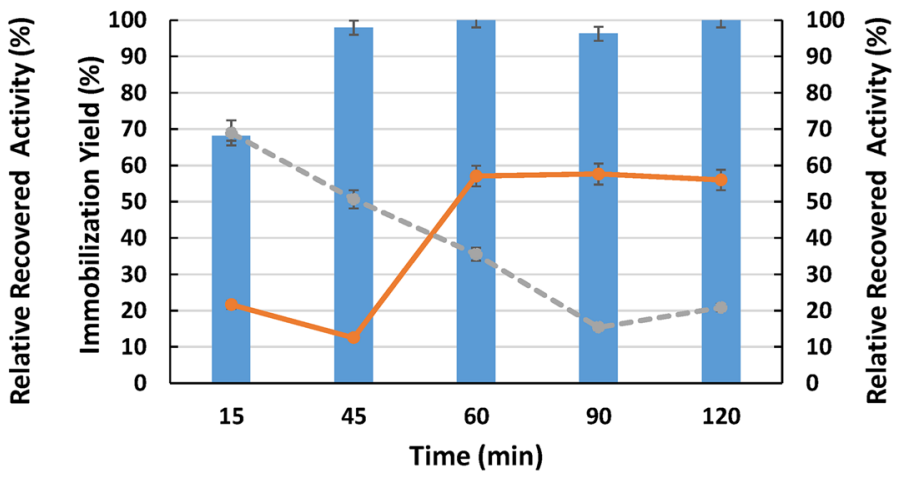

Figure 2. Effect of contact time on immobilization yield (columns) and relative recovered activity (orange lines) obtained with the immobilization of $8.6 \times 10^{-5} \mathrm{~g} \mathrm{~mL}^{-1}$ of ASNase on $2 \mathrm{mg}$ of functionalized MWCNTs (functionalization with $\mathrm{HNO}_{3}$ aqueous solutions with variable concentrations: 0.05, 0.10, 0.20 and $0.30 \mathrm{M}$ ) at $\mathrm{pH}$ 8. Grey lines represent the relative recovered activity of ASNase on $2 \mathrm{mg}$ of MWCNTs without any functionalization (MWCNT-NC, control).

Contact time effect on ASNase immobilization. The influence of contact time on the ASNase immobilization was also addressed using the selected $\mathrm{pH}$ of 8 and all modified MWCNTs. Five contact times, between 15 and 120 min were evaluated. The results presented in Fig. 2 (detailed data in Table S2 in the Supporting Information) reveal that contact time significantly affects the ASNase immobilization yield and RRA. The contact time of 15 min promotes the lowest values of immobilization yield $\left(\sim 70 \%, \sim 1.20 \times 10^{-5} \mathrm{~g}\right.$ of ASNase was immobilized) for all MWCNTS used. These results may be due to the short period of contact between the enzyme and the MWCNTS, not allowing the complete attachment of ASNase. On the other hand, after 60 min of contact time, the highest values of immobilization yield were reached (higher than $90 \%$, more than $1.55 \times 10^{-5} \mathrm{~g}$ of ASNase was immobilized) for all the MWCNTS.

Regarding the RRA, the same behavior was observed, 15 min of contact time was not enough to obtain high ASNase RRA values for all MWCNTs studied (12-37\%). However, after $60 \mathrm{~min}$, maximum ASNase RRA values were attained (58-69\%), except for MWCNT-NC-0.05, which needs 90 min to attain 56\% of RRA, probably due to a lower extent of surface functionalization. Among the functionalized MWCNTs, the best results were obtained with MWCNT-NC-0.1 (as previously reported for the assays regarding the $\mathrm{pH}$ effect). Comparing to non-functionalized MWCNTs, the previous results with surface-modified MWCNTs exhibit an opposite behavior (Fig. 2-grey lines).

It is clear that the physical and chemical properties of the MWCNTs influence the catalytic performance of ASNase. The RRA of ASNase immobilized in pristine MWCNTs decreased with the increase in contact time, suggesting that the oxidative modification of MWCNTs has a beneficial protector effect on the ASNase RRA. It is recognized that the acid oxidation treatment modifies the physical and chemical properties of CNTs, leading to an improvement of CNTs biocompatibility ${ }^{27}$. In the present work, when MWCNTs are oxidized, their hydrophilicity increases due to the introduction of oxygen-containing functional groups, i.e., carboxyl, carbonyl, and phenol groups, as described below. This surface modification may promote a proper interaction between the enzyme and the nanomaterial, thus leading to an increase in the RRA of ASNase ${ }^{36}$. Besides, metal catalyst particles present in the surface of MWCNTs, which can inhibit the ASNase activity, are removed during the acidic treatment ${ }^{37}$. Considering immobilization yields of $100 \%$ and RRA values higher than $50 \%, 60$ min of contact time was chosen for further studies.

Enzyme concentration effect on ASNase immobilization. The ASNase concentration and materials adsorption capacity were finally investigated at the optimized $\mathrm{pH}$ and contact time ( $\mathrm{pH} 8.0$ and $60 \mathrm{~min}$ ) for all MWCNTs. 
MWCNT-NC-0.05

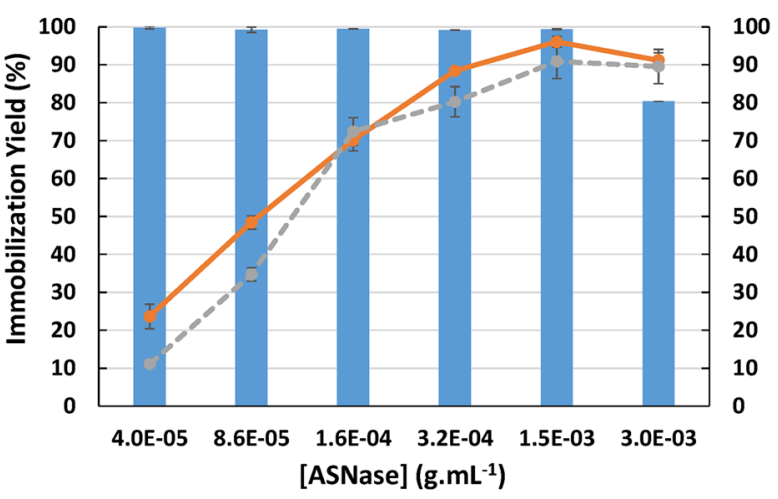

MWCNT-NC-0.2

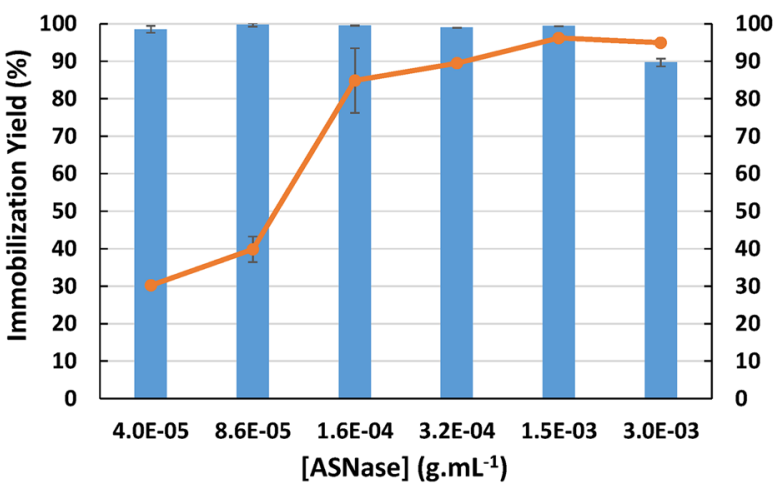

MWCNT-NC-0.1

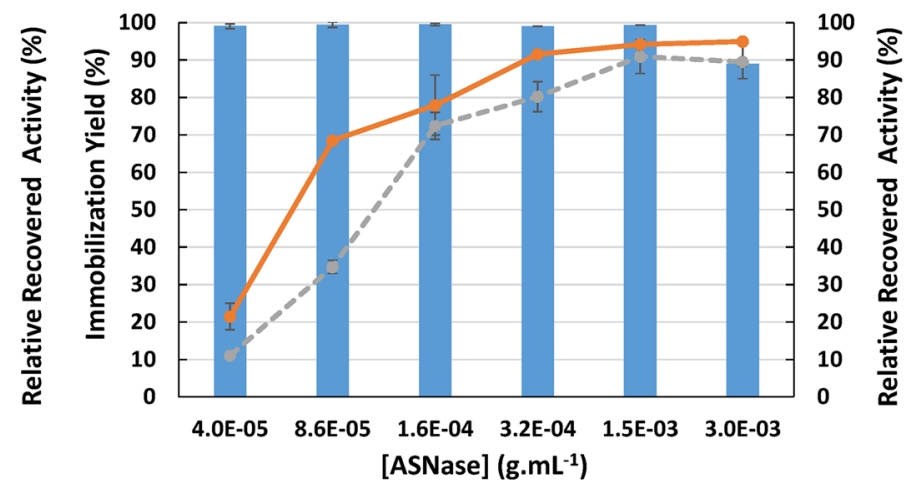

MWCNT-NC-0.3

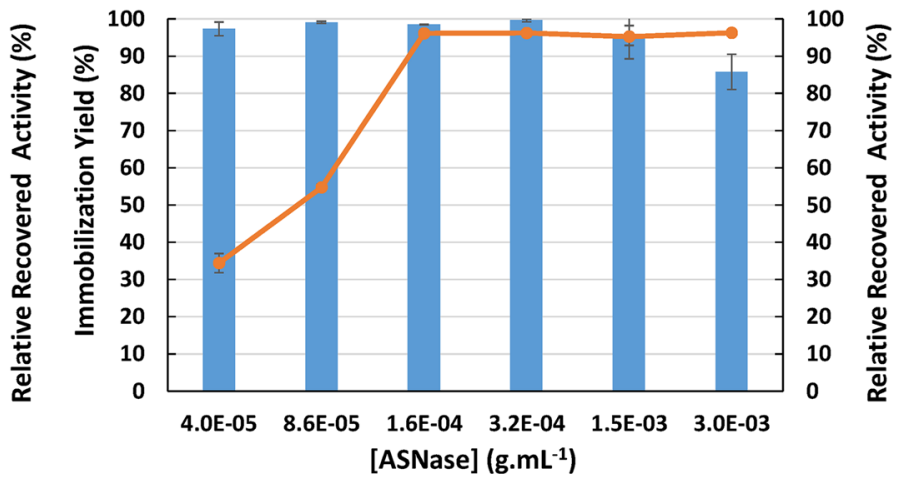

Figure 3. Effect of ASNase concentration on immobilization yield (columns) and relative recovered activity (orange lines) obtained with the immobilization of ASNase on $2 \mathrm{mg}$ of functionalized MWCNTs (functionalization with $\mathrm{HNO}_{3}$ aqueous solutions with variable concentrations: $0.05,0.10,0.20$ and $0.30 \mathrm{M}$ ) at $\mathrm{pH} 8$ during $60 \mathrm{~min}$. Grey lines represent the relative recovered activity of ASNase on $2 \mathrm{mg}$ of MWCNTs without any functionalization (MWCNT-NC, control).

Six enzyme concentrations ranging between $4.0 \times 10^{-5} \mathrm{~g} \mathrm{~mL}^{-1}\left(8.00 \times 10^{-6} \mathrm{~g}\right.$ of ASNase $)$ and $3.0 \times 10^{-3} \mathrm{~g} \mathrm{~mL}^{-1}$ $\left(6.00 \times 10^{-4} \mathrm{~g}\right.$ of ASNase) were studied. The results obtained are presented in Fig. 3 (detailed data in Table S3 in the Supporting Information). Regarding the ASNase immobilization yield, it is possible to observe complete adsorption (100\% of enzyme immobilization yield) for almost all the enzyme concentrations studied and for all the materials tested. Only when the highest concentration of enzyme studied was evaluated $\left(3.0 \times 10^{-3} \mathrm{~g} \mathrm{~mL}^{-1}\right.$; $6.00 \times 10^{-4} \mathrm{~g}$ of ASNase), a decrease in the immobilization yield was observed, but still higher than $80 \%$ (more than $2.40 \times 10^{-3} \mathrm{~g}$ of ASNase was immobilized), suggesting that the maximum adsorption capacity was attained with a ASNase concentration of $1.5 \times 10^{-3} \mathrm{~g} \mathrm{~mL}^{-1}\left(3.00 \times 10^{-4} \mathrm{~g}\right.$ of ASNase) for all the MWCNTs used. The ASNase RRA increases with ASNase concentration up to $1.5 \times 10^{-3} \mathrm{~g} \mathrm{~mL}^{-1}$, corresponding to values higher than $90 \%$ of RRA. An exception is observed when using the MWCNT-NC 0.3 , where the maximum RRA (higher than $95 \%)$ was achieved using an ASNase concentration of $1.6 \times 10^{-4} \mathrm{~g} \mathrm{~mL}^{-1}\left(3.20 \times 10^{-5} \mathrm{~g}\right.$ of ASNase). This same behavior was observed for non-functionalized MWCNTs; however, the RRA values were lower when compared to modified MWCNTs, as explained above.

Similar ASNase immobilization yield (97.0\%) was reported in the literature using calcium-alginate/MWCNTs hybrid beads $\mathrm{s}^{30}$, although the immobilization approach used in our work may be considered more straightforward. On the other hand, Haroun et al. ${ }^{31}$ used a similar strategy to the one used in this work and applied oxidized MWCNTs to immobilize ASNase, but lower values of immobilization yield (54.4\%) were achieved.

Adsorption isotherm studies were carried out to better understand the ASNase adsorption behavior over the MWCNTs. The experimental data were fitted to the Langmuir and Freundlich models (Eqs. 5 and 6, respectively). Taking into account the correlation coefficient $\left(R^{2}\right)$, the experimental equilibrium data were better fitted to the Langmuir model, with a $R^{2}$ ranging between 0.90 and 0.94 , while for the Freundlich model a $R^{2}$ ranging between 0.72 and 0.80 was determined for the functionalized MWCNTs (Figure S2 and detailed data in Table S4 given in the Supporting Information). Similar results were described by Cristóvão et al. ${ }^{29}$ for non-functionalized MWCNTS. These results disclose the absence of ASNase multilayers formation on the support.

A good fit between the Langmuir model predictions and the experimental data (adsorption of several ASNase concentrations onto functionalized MWCNTs at $\mathrm{pH} 8$ for $60 \mathrm{~min}$ ) was observed (Fig. S2), indicating the formation of an ASNase monolayer on the support surface. The maximum ASNase adsorption capacity $\left(q_{\max }\right)$ values are similar for all the functionalized MWCNTs studied: 152.5, 155.7, 156.3 and $157.6 \mathrm{U} \mathrm{g}^{-1}$ for MWCNT-NC-0.05, MWCNT-NC-0.1, MWCNT-NC-0.2 and MWCNT-NC-0.3, respectively. A slight increase in the adsorption 


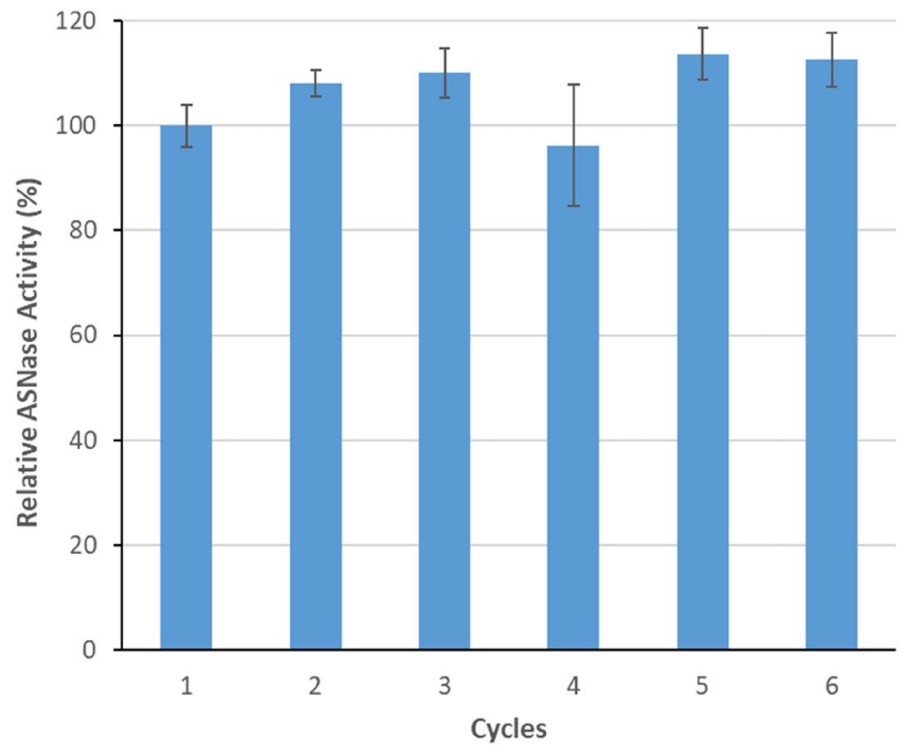

Figure 4. Operational stability of immobilized ASNase onto MWCNT-NC-0.3 during 60 min of contact time, $\mathrm{pH} 8.0$, and with $1.5 \times 10^{-3} \mathrm{~g} \mathrm{~mL}^{-1}$ of initial ASNase concentration.

\begin{tabular}{|l|l|l|l|l|l|l|}
\hline & $\mathbf{S}_{\text {BET }}\left(\mathbf{m}^{2} \mathbf{g}^{-1}\right)$ & {$[\mathbf{C O}]\left(\mathbf{m m o l ~ g}^{-1}\right)$} & {$\left[\mathbf{C O}_{2}\right]\left(\mu \mathbf{m o l ~ g}^{-1}\right)$} & {$[\mathbf{C O}]+\left[\mathbf{C O}_{2}\right]$} & $\left.[\mathbf{C O}] /] \mathbf{C O}_{2}\right]$ & $\mathbf{O}(\%)$ \\
\hline MWCNT & 189 & 95 & 25 & 120 & 3.80 & 0.25 \\
\hline MWCNT-NC-0.05 & 227 & 145 & 45 & 190 & 3.22 & 1.4 \\
\hline MWCNT-NC-0.1 & 262 & 903 & 291 & 1194 & 3.10 & 1.7 \\
\hline MWCNT-NC-0.2 & 271 & 1266 & 402 & 1668 & 3.15 & 2.4 \\
\hline MWCNT-NC-0.3 & 278 & 1541 & 489 & 2030 & 3.15 & 2.9 \\
\hline
\end{tabular}

Table 1. Surface area $\left(\mathrm{S}_{\mathrm{BET}}\right)$, concentrations of $\mathrm{CO}$ and $\mathrm{CO}_{2}$ obtained by TPD analysis and oxygen content obtained from elemental analysis.

capacity for all the functionalized nanomaterials was observed compared with the pristine MWCNTs $\left(q_{\max }\right.$ for ASNase of $148.0 \mathrm{U} \mathrm{g}^{-1}$ ).

Operational stability of ASNase-MWCNTs bioconjugate. Envisioning the industrial application of ASNaseMWCNTs in food or therapeutic sectors, the reusability of the bioconjugate is mandatory. This issue was studied by analyzing several cycles of L-asparagine hydrolysis using the most promising nanomaterial-MWCNTNC-0.3 for the immobilization of $1.5 \times 10^{-3} \mathrm{~g} \mathrm{~mL}^{-1}$ of ASNase. The results provided in Fig. 4 show that, despite using a simple ASNase adsorption method (where relatively weak interactions are expected to be involved), the overall system reveals exceptional operational stability, without any loss of immobilized ASNase activity during 6 consecutive reaction cycles. These results also emphasize the improved ASNase bioconjugate stability acquired after attachment onto functionalized MWCNTs. This confirmed stability is a relevant acquired advantage, allowing bioconjugate reuse several times in batch processes, as well as the application in continuous processing systems. Other authors have studied the immobilized ASNase operational stability during successive cycles using different supports; nevertheless, some activity losses have been reported. For example, Noma et al..$^{38}$ reported $88 \%$ of ASNase initial activity after 5 reuse cycles when immobilized on $\mathrm{Fe}_{3} \mathrm{O}_{4} / \mathrm{S}_{\mathrm{i}} \mathrm{O}_{2} / \mathrm{NH}_{2}$ and $\mathrm{Fe}_{3} \mathrm{O}_{4} / \mathrm{COOH}$ particles. In turn, Orhan and Uygun ${ }^{39}$ demonstrated that an ASNase-magnetic nanoparticles bioconjugate maintained $85.14 \%$ of its initial activity after 5 successive cycles. In the current work, with the ASNase-MWCNTs there are no losses on the immobilized enzyme activity for 6 cycles of reuse and reaction.

ASNase-MWCNTs bioconjugate characterization. The texture and surface chemistry of pristine and functionalized MWCNTs were investigated to obtain an insight into the extent and nature of the interactions between the MWCNTs and ASNase (Table 1). This information is essential to better understand the improved enzyme immobilization yield and the respective RRA with the ASNase-MWCNTs bioconjugate.

The acid treatment is known to introduce defects into the sidewalls of the MWCNTs as well as open up the end caps of the tubes (Fig. $5 \mathrm{a})^{40}$, leading to an increase in the surface area $\left(\mathrm{S}_{\mathrm{BET}}\right)$ of the resulting materials. As the concentration of $\mathrm{HNO}_{3}$ used in the oxidation treatment increases, a progressive increase in the $S_{\mathrm{BET}}$ of the 

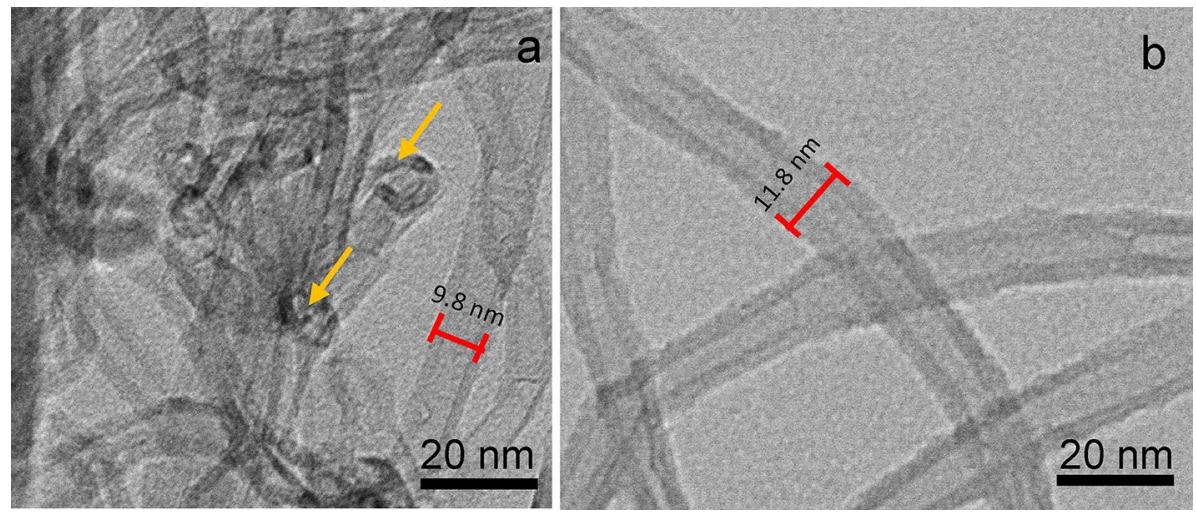

Figure 5. TEM micrographs of MWCNT-NC-0.3 (a) before and (b) after ASNase immobilization. The yellow arrows are marking the opened ends of the MWCNTs.

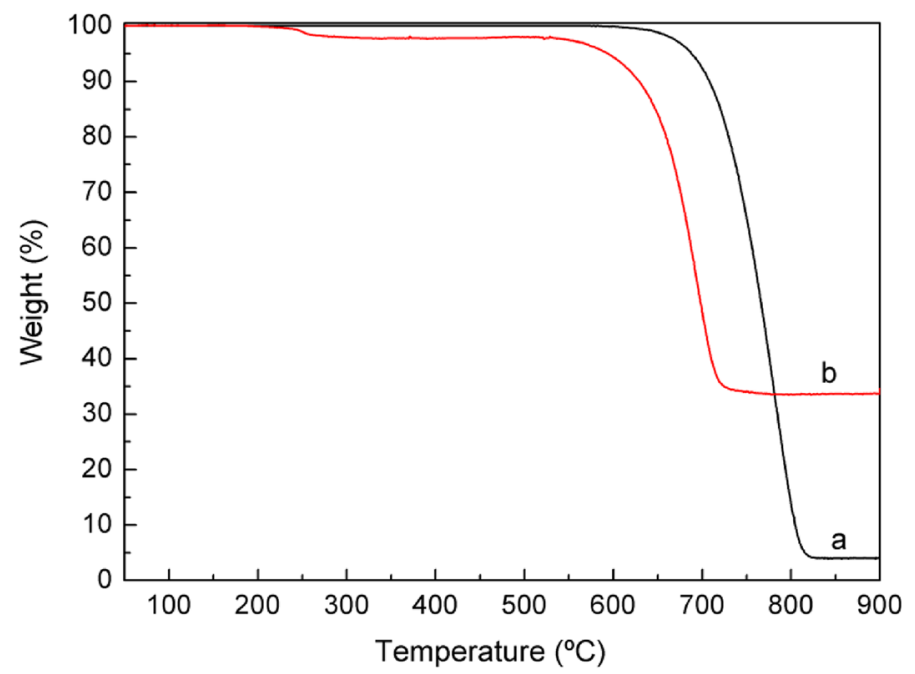

Figure 6. TGA analysis of MWCNT-NC-0.3 (a) before and (b) after ASNase immobilization.

materials is observed (Table 1), which may be related to the improved ASNase adsorption ability of the MWCNTs. The $\mathrm{S}_{\mathrm{BET}}$ varied from $189 \mathrm{~m}^{2} \mathrm{~g}^{-1}$ to $278 \mathrm{~m}^{2} \mathrm{~g}^{-1}$ for unmodified MWCNTs and MWCNT-NC-0.3, respectively.

The treatment with $\mathrm{HNO}_{3}$ produces materials with large quantities of surface oxygen groups, mainly carboxylic acids and, to a smaller extent, lactones, anhydrides, and phenol groups formed at the edges/ends and defects of graphitic sheets ${ }^{41-43}$. These groups are decomposed by heating the MWCNTs samples and are released in the form of $\mathrm{CO}$ and/or $\mathrm{CO}_{2}$, which were followed by temperature-programmed desorption (TPD) analysis (Figures S3 and S4 in Supporting Information). The total amounts of $\mathrm{CO}$ and $\mathrm{CO}_{2}$ released from the various CNT samples, obtained by integration of the TPD spectra, are presented in Table 1 . The total amount of oxygen surface groups (released as $\mathrm{CO}$ and $\mathrm{CO}_{2}$ ) increase with the concentration of $\mathrm{HNO}_{3}$ used for the oxidation treatment, as can also be appraised by the percentage of oxygen determined by elemental analysis (Table 1). Moreover, the lower $\mathrm{CO} / \mathrm{CO}_{2}$ ratio observed for the oxidized MWCNTs, compared with the pristine material, indicates a more acidic surface, which is mainly due to the presence of carboxylic acid groups.

The immobilization of $1.5 \times 10^{-3} \mathrm{~g} \mathrm{~mL}^{-1}$ of ASNase over the MWCNTs was also confirmed by TEM, as displayed in Fig. 5 for MWCNT-NC-0.3 and ASNase-MWCNT-NC-0.3. Typical tubular morphology of MWCNTs was observed as well as the opened ends of the tubes (marked with arrows in Fig. 5a). After ASNase immobilization, the walls of the MWCNTs become thicker and more irregular due to the presence of the enzyme at the surface of the carbon nanomaterial (Fig. 5b). The difference between the thickness of the naked nanotubes and the ASNase-MWCNT-NC-0.3 bioconjugate gives an estimated thickness of the enzyme film of $2 \mathrm{~nm}$.

Thermogravimetric analysis (TGA) of neat MWCNT-NC-0.3 and the respective ASNase bioconjugate, ASNase-MWCNT-NC-0.3 (immobilization with $1.5 \times 10^{-3} \mathrm{~g} \mathrm{~mL}^{-1}$ of ASNase per $2 \mathrm{mg}$ of material), is presented in Fig. 6. TGA results reveal that the MWCNT-NC-0.3 material starts to decompose at around $580^{\circ} \mathrm{C}$ and is completely burned at $820^{\circ} \mathrm{C}$. At this temperature, a plateau is reached, corresponding to $3.9 \%$ of the initial mass, attributed to the presence of ashes resulting from the pyrolysis of inorganic impurities. Differently, two main weight losses are observed for the ASNase-MWCNT-NC-0.3 bioconjugate. The first weight loss is detected at c.a. $250{ }^{\circ} \mathrm{C}$, which is attributed to the thermal decomposition of the enzyme ${ }^{44}$, while the second weight loss at $600{ }^{\circ} \mathrm{C}$ 


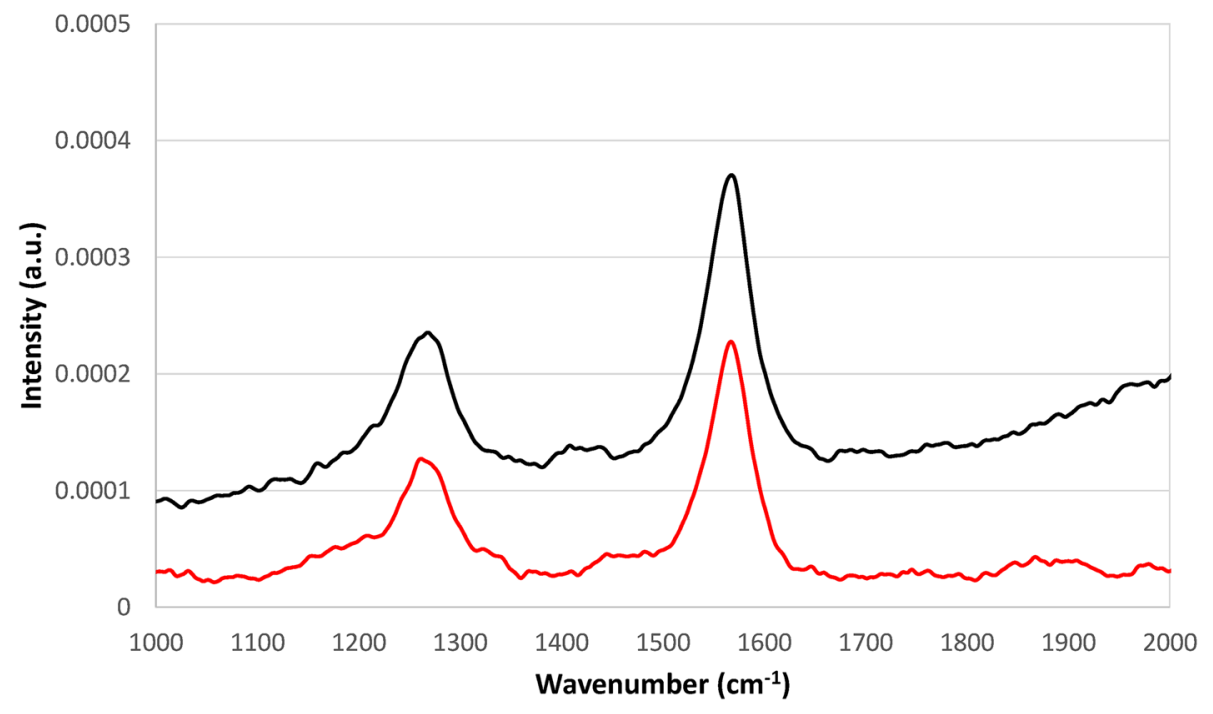

Figure 7. Raman spectra of MWCNT-NC-0.3 (black line) and ASNase-MWCNT-NC-0.3 bioconjugate (red line).

is recognized as the simultaneous pyrolysis of ASNase and MWCNTs, attaining a plateau at a temperature near to $700{ }^{\circ} \mathrm{C}$. This plateau corresponds to $33.8 \%$ of the initial mass of the enzyme-support complex. These results suggest that the enzyme content of the bioconjugate should be c.a. $29.7 w t$. \% (difference between the weight loss obtained for the neat MWCNT-NC- 0.3 and the bioconjugate), which corresponds to $0.422 \mathrm{~g}$ of enzyme per $\mathrm{g}$ of carbon nanotubes.

Raman spectroscopy was also used for characterizing the surface of the MWCNT-NC-0.3 before and after immobilization of $1.5 \times 10^{-3} \mathrm{~g} \mathrm{~mL}^{-1}$ of ASNase. The Raman spectra exhibit two prominent characteristic bands in the spectral region between 1000 and $2000 \mathrm{~cm}^{-1}$ (Fig. 7). The $\mathrm{G}$ band, peaking at c.a. $1600 \mathrm{~cm}^{-1}$, corresponds to the MWCNTs regular sp ${ }^{2}$ graphitic network and is common to both materials, while the D band at $1287 \mathrm{~cm}^{-1}$ is assigned to the disorder and defects caused by $\mathrm{sp}^{3}$ hybridized carbon in the lattice ${ }^{45}$. The ratio between the intensities of $\mathrm{D}$ and $\mathrm{G}$ bands $\left(\mathrm{I}_{\mathrm{D}} / \mathrm{I}_{\mathrm{G}}\right)$ gives information about the degree of disorder and the existence of defect sites in the MWCNTs lattice ${ }^{46}$. The $\mathrm{I}_{\mathrm{D}} / \mathrm{I}_{\mathrm{G}}$ values obtained for MWCNT-NC-0.3 and ASNase-MWCNT-NC- 0.3 were 0.636 and 0.631 , respectively. These similar values suggest that the enzyme immobilization in the nanomaterials do not cause a significant disturbance on the CNTs surface, indicating similar degrees of disorder, i.e., ASNase adsorption occurs predominantly on the defect sites present at the MWCNT-NC-0.3 surface, not changing the $\mathrm{sp}^{2}$ and $\mathrm{sp}^{3}$ bonds concentration.

\section{Conclusions}

The hydrothermal functionalization of MWCNTs significantly improves the interaction with the ASNase enzyme. The MWCNTs functionalized with $0.3 \mathrm{M} \mathrm{HNO}_{3}$ aqueous solution produced the highest immobilization yield and relative recovered activity of ASNase above 95\%. With these levels of immobilization and activity, one can unlock higher stages of application for this ASNase-MWCNT bioconjugate, like new drug carriers development for concrete targets (e.g., aggressive cancer types) and a wider application in the food industry.

Tunning of the process could be done by controlling the $\mathrm{pH}$ value, contact time between the enzyme and the support, and ASNase/MWCNTs mass ratio during the immobilization procedure. The ASNase/MWCNTs mass ratio was the parameter with the most substantial effect on improving immobilization.

The optimized adsorption conditions (using the Langmuir model) to achieve an immobilization yield and a relative recovery activity of ASNase above $95 \%$ consisted of $\mathrm{pH} 8.0,60 \mathrm{~min}$ of contact time and enzyme concentration of $1.5 \times 10^{-3} \mathrm{~g} \mathrm{~mL}^{-1}$ in $2.0 \mathrm{mg}$ of MWCNTs. These conditions correspond to an MWCNT maximum predicted enzyme loading capacity of $158.0 \mathrm{U} \mathrm{g}^{-1}$. Because the adsorption results followed well a Langmuir isotherm behavior, it can be assumed that the adsorption of an ASNase on the support consists of a monolayer. It results in excellent operational stability, with the ASNase immobilization onto MWCNTs without any ASNase activity loss for 6 reaction cycles. Enzymatic activity and stability, which are dependent on the oxidized MWCNT surfaces, types of interactions formed and concentrations used, resulted in exceptional operational stabilities under optimized conditions. This demonstrates the potential for improved industrial applications, where the prepared catalyst can be easily separated and reused several times, reducing the demand for the enzyme and improving the economy of the process. All of these advantages outweigh the technical requirements needed to modify the MWCNTs.

In general, the provided straightforward approach demonstrates how functionalization of MWCNTs can be tailored to support ASNase, or any other enzyme efficiently, opening the possibility to produce specific bioconjugates for several application fields, e.g., as biosensors and the pharmaceutical and food industry. 


\section{Experimental section}

Materials. Lyophilized ASNase (with no additives; purity >96.0\%) from E. coli ASI.357 (ENZ-287) was supplied by Prospec. Multi-walled carbon nanotubes (MWCNTs) with average diameter and length of $9.5 \mathrm{~nm}$ and $1.5 \mu \mathrm{m}$, respectively, specific surface area $\left(\mathrm{S}_{\mathrm{BET}}\right)$ of $189 \mathrm{~m}^{2} \mathrm{~g}^{-1}$ and purity $\geq 95 \%$ were purchased from Nanocyl (NC3100). Nitric acid used for MWCNTs functionalization was supplied by Sigma-Aldrich. L-asparagine $(\geq 99.0 \%)$ was supplied by Acros Organics. Disodium phosphate heptahydrate (98.0-102\%), sodium carbonate (99.0\%), and sodium hydrogen carbonate (99.5\%) were acquired from Sigma-Aldrich, Vencilab and Prolabo, respectively. Tris(hydroxymethyl)aminomethane (TRIS) ( $\geq 99.8 \%$ ), trichloroacetic acid (TCA) (Analytic), and Nessler's reagent were purchased from Prolabo, Prunella and Fluka, respectively.

MWCNTs functionalization. Traditional methods for the introduction of oxygen functionalities at the surface of carbon materials include hydrothermal oxidation with nitric and sulphuric acids, and with hydrogen peroxide, and also thermal treatment with oxygen in gas phase. In previous works on the immobilization of other enzymes, such as laccase and peroxidase over MWCNTs, we found that the presence of hydroxyl groups are beneficial for improving the interaction of the proteins with the surface of the carbon nanotubes ${ }^{21,22}$. In those works, the functionalization consisted in the oxidation of the MWCNTs by heating at reflux in a highly concentrated $\mathrm{HNO}_{3}$ aqueous solution $(7 \mathrm{M})$, followed by washing with distilled water until neutral $\mathrm{pH}$. In fact, that process generated high volumes of residual rinsing water containing nitric acid. Therefore, in the present work, we used an alternative approach consisting of a high-pressure hydrothermal treatment, which allowed the use of much less concentrated nitric acid solutions $(0.1-0.3 \mathrm{M})$ and half the volume of solution to treat the same amount of MWCNTs ${ }^{12,47}$. Moreover, the amount of oxygen functionalities introduced at the surface of the carbon nanomaterials was similar using both techniques.

In this work, hydrothermal oxidation of the pristine MWCNTs was carried out in a Teflon-lined stainless-steel autoclave using $\mathrm{HNO}_{3}$ aqueous solutions with variable concentrations $(0.05,0.10,0.20$ and $0.30 \mathrm{M})$ at $200{ }^{\circ} \mathrm{C}$, as described elsewhere ${ }^{12}$. Briefly, $0.2 \mathrm{~g}$ of MWCNTs was added to $75 \mathrm{~mL}$ of an $\mathrm{HNO}_{3}$ aqueous solution. After being sealed, the vessel was put into an oven at $200^{\circ} \mathrm{C}$ for $2 \mathrm{~h}$. Then, the MWCNTs were recovered, rinsed with water until neutrality, and dried overnight at $120^{\circ} \mathrm{C}$. The resulting materials are labeled as MWCNT-NC-Y, where $\mathrm{Y}$ corresponds to the $\mathrm{HNO}_{3}$ concentration (in $\mathrm{M}$ ) used in the oxidation treatment $(\mathrm{Y}=0.05,0.10,0.20$ and $0.30 \mathrm{M})$. These materials were used for the subsequent ASNase immobilization.

ASNase immobilization on functionalized MWCNTs. The ASNase immobilization over the functionalized MWCNTs was carried out by direct physical adsorption in batch system. Several parameters were studied to maximize the ASNase adsorption, namely $\mathrm{pH}$, contact time and ASNase/MWCNT mass ratio, and reported in terms of immobilization yield and relative recovered ASNase activity. In a typical experiment, MWCNTs $(2.0 \pm 0.1 \mathrm{mg})$ were added to $200 \mu \mathrm{L}$ of an ASNase solution, and the suspensions were left in contact in an orbital stirrer, as detailed below.

The $\mathrm{pH}$ effect on the ASNase adsorption and relative recovered activity were studied using a $0.15 \mathrm{M}$ citrate-phosphate buffer at $\mathrm{pH}$ value 5.0 and a $0.2 \mathrm{M}$ phosphate buffer at $\mathrm{pH}$ values of 6.0, 7.0 and 8.0. For that purpose, $200 \mu \mathrm{L}$ of ASNase (final concentration of $8.6 \times 10^{-5} \mathrm{~g} \mathrm{~mL}^{-1} ; 1.72 \times 10^{-5} \mathrm{~g}$ of ASNase) was prepared in each studied buffer and added to the MWCNTs. This mixture was stirred for $60 \mathrm{~min}$ in an orbital shaker at $50 \mathrm{rpm}$. A control was also prepared using free ASNase at $8.6 \times 10^{-5} \mathrm{~g} \mathrm{~mL}^{-1}\left(1.72 \times 10^{-5} \mathrm{~g}\right.$ of ASNase $)$ in each of the evaluated buffers.

The influence of the contact time between the ASNase and the MWCNTs was also investigated. The experiments were performed by adding $200 \mu \mathrm{L}$ of ASNase $8.6 \times 10^{-5} \mathrm{~g} \mathrm{~mL}^{-1}\left(1.72 \times 10^{-5} \mathrm{~g}\right.$ of ASNase $)$ in $0.2 \mathrm{M}$ phosphate buffer at $\mathrm{pH} 8.0$ to the MWCNTs. The $\mathrm{pH} 8.0$ was selected, taking into account the results obtained in the previous assays. The mixture was stirred during periods ranging between 15 and $120 \mathrm{~min}$ in an orbital shaker at $50 \mathrm{rpm}$. A control sample was prepared using $200 \mu \mathrm{L}$ of ASNase $8.6 \times 10^{-5} \mathrm{~g} \mathrm{~mL}^{-1}\left(1.72 \times 10^{-5} \mathrm{~g}\right.$ of ASNase $)$ in phosphate buffer at $\mathrm{pH} 8.0$.

Finally, the effect of ASNase concentration was evaluated by adding $200 \mu \mathrm{L}$ of different ASNase concentrations, from $4.0 \times 10^{-5}\left(8.00 \times 10^{-6} \mathrm{~g}\right.$ of ASNase $)$ to $3.0 \times 10^{-3}\left(6.00 \times 10^{-4} \mathrm{~g}\right.$ of ASNase $) \mathrm{g} \mathrm{mL}^{-1}$, at $\mathrm{pH} 8.0$, to the MWCNTs. The mixture was stirred for $60 \mathrm{~min}$ in an orbital shaker. Both $\mathrm{pH}$ value and contact time were selected, taking into account the results obtained in the previous assays. A control was prepared with $200 \mu \mathrm{L}$ of ASNase in phosphate buffer at $\mathrm{pH} 8.0$ at each concentration.

At least three individual experiments were carried out for each condition, allowing the determination of the average immobilization yield and relative recovered activity and respective standard deviations.

ASNase activity determination. The enzyme activity was determined by quantifying ammonia released after the L-asparagine (substrate) hydrolysis by ASNase. The experimental procedure comprises the mixture of $50 \mu \mathrm{L}$ of $\mathrm{L}$-asparagine $(189 \mathrm{mM})$ with $50 \mu \mathrm{L}$ of enzyme solution (initial free enzyme or supernatant after immobilization) or with $2.0 \mathrm{mg}$ of attached ASNase on MWCNTS (immobilized enzyme) in $500 \mu \mathrm{L}$ of TRIS-HCl buffer $(50 \mathrm{mM}$ and $\mathrm{pH} 8.6)$, and $450 \mu \mathrm{L}$ of deionized water, at $37^{\circ} \mathrm{C}$ for $30 \mathrm{~min}$ under stirring. After incubation, for the free enzyme, the reaction was stopped by adding $250 \mu \mathrm{L}$ of $1.5 \mathrm{M}$ TCA, while for the immobilized enzyme, the supernatant (containing ammonia) was separated throughout centrifugation at $12000 \mathrm{rpm}$ during $15 \mathrm{~min}$. Subsequently, $100 \mu \mathrm{L}$ of the previous free enzyme solution or $100 \mu \mathrm{L}$ of the supernatant was mixed with $2.15 \mathrm{~mL}$ of deionized water and $250 \mu \mathrm{L}$ of Nessler's reagent to measure the ammonia amount ${ }^{48}$. After 30 min of incubation, the increase in absorbance was measured by spectroscopy, using a BioTeck Synergy HT microplate reader at $436 \mathrm{~nm}$. A calibration curve was previously established using ammonium sulfate. Possible interferences of CNTs with Nessler's reagent were investigated, and no interference was found. 
One unit of free ASNase activity is defined as the amount of enzyme that releases $1 \mu \mathrm{mol}$ of ammonia per minute at $37^{\circ} \mathrm{C}$ (Eq. 1):

$$
\text { ASNase activity }\left(\frac{U}{L}\right)=\frac{\left[N H_{4}^{+}\right]\left(\frac{\mu m o l}{m L}\right) \times V_{\text {Nessler }}(m L) \times f_{d}}{t_{r}(\min )}
$$

where $V_{\text {Nessler }}$ is the volume of the Nessler solution, $f_{d}$ is the sample dilution factor, and $t_{r}$ is the reaction time.

One unit of immobilized ASNase activity is defined as the amount of enzyme that releases $1 \mu \mathrm{mol}$ of ammonia per minute and mass of support at $37^{\circ} \mathrm{C}$ (Eq. 2).

$$
\text { ASNase activity }\left(\frac{U}{m g}\right)=\frac{\left[N H_{4}^{+}\right]\left(\frac{\mu m o l}{m L}\right) \times V_{\text {Nessler }}(m L) \times f_{d}}{t_{r}(\mathrm{~min}) \times m_{s}(m g)}
$$

where $m_{s}$ is the mass of the support.

The immobilization yield, $I Y(\%)$, is defined as the difference between the free enzyme activity before immobilization and the activity of the free enzyme remaining in the supernatant after immobilization divided by the free enzyme activity before immobilization (Eq. 3).

$$
I Y(\%)=\frac{\text { Free ASNase Activity }\left(\frac{U}{m L}\right)-\text { Supernatant ASNase Activity }\left(\frac{U}{m L}\right)}{\text { Free ASNase Activity }\left(\frac{U}{m L}\right)} \times 100
$$

The relative recovered activity, $R R A(\%)$, of the immobilized enzyme, was calculated as the ratio between the activity of the effectively immobilized enzyme and the maximum theoretical activity that would exist if the free enzyme was totally immobilized (Eq. 4).

$$
R R A(\%)=\frac{\text { Immobilized ASNase activity }\left(\frac{U}{m g}\right)}{\text { Maximum ASNase activity }\left(\frac{U}{m g}\right)}
$$

where: Maximum ASNase activity $\left(\frac{U}{m g}\right)=\frac{\left[N H_{4}^{+}\right]_{\text {freeASNase }}\left(\frac{\mu m o l}{m L}\right) \times V_{\text {Nessler }}(m L) \times f_{d}}{t_{r}(\min ) \times m_{s}(m g)}$

Adsorption isotherms. The adsorption equilibrium behavior of the ASNase onto MWCNTs was evaluated by adjusting the experimental results to the Langmuir and Freundlich isotherm models. The ASNase concentrations were varied from $4.0 \times 10^{-5}\left(8.00 \times 10^{-6} \mathrm{~g}\right.$ of ASNase $)$ to $3.0 \times 10^{-3}\left(6.00 \times 10^{-4} \mathrm{~g}\right.$ of ASNase $) \mathrm{g} \mathrm{mL}^{-1}$ at $\mathrm{pH} 8.0$, and the equilibrium time was fixed at $60 \mathrm{~min}$.

The Langmuir model parameters were determined by fitting Eq. 5 to the experimental data using the CurveExpert v 1.38 software:

$$
q=\frac{q_{\max } \times K \times C}{1+K \times C}
$$

where $q$ is the amount of adsorbed active ASNase $(\mathrm{U})$ per gram of MWCNT $\left(\mathrm{U} \mathrm{g}^{-1}\right), q_{\max }$ is the maximum adsorption capacity of active ASNase $\left(\mathrm{U} \mathrm{g}^{-1}\right), K$ is the Langmuir adsorption equilibrium constant $\left(\mathrm{mL} \mathrm{g}^{-1}\right)$ related to the strength of affinity between the protein and the surface ${ }^{49}$ and $C$ is the ASNase concentration $\left(\mathrm{g} \mathrm{mL}^{-1}\right)$.

The Freundlich model parameters were estimated by fitting of Eq. (6) to the experimental data using the CurveExpert v 1.38 software:

$$
q=k_{F} \times C^{1 / n}
$$

where $k_{F}$ is the Freundlich binding constant related to the adsorption of ASNase per weight of MWCNT $\left[\left(\mathrm{U} \mathrm{g}^{-1}\right) \cdot\left(\mathrm{mL} \mathrm{g}^{-1}\right)^{(1 / \mathrm{n})}\right]$, and $n$ is an empirical parameter that is a measure of the intensity of adsorption in Freundlich adsorption isotherms.

Operational stability of immobilized ASNase. The operational stability of ASNase-MWCNTs bioconjugate was assessed by incubating $2 \mathrm{mg}$ of the immobilized ASNase with $50 \mu \mathrm{L}$ of L-asparagine $(189 \mathrm{mM})$ in 500 $\mu \mathrm{L}$ of TRIS- $\mathrm{HCl}$ buffer ( $50 \mathrm{mM}$ and $\mathrm{pH} 8.6)$, and $450 \mu \mathrm{L}$ of deionized water, at $37^{\circ} \mathrm{C}$ for 30 min under stirring. At the end of each cycle, the reaction was stopped by the supernatant removal throughout centrifugation at 12 $000 \mathrm{rpm}$ for $15 \mathrm{~min}$, and subsequent addition of $250 \mu \mathrm{L}$ of TCA $1.5 \mathrm{M}$ in the supernatant. The immobilized ASNase was washed twice with phosphate buffer $\mathrm{pH}$ 8.0 $( \pm 500 \mu \mathrm{L}$ each wash $)$ and resuspended in a fresh substrate solution to begin the next cycle. Six cycles of operational stability were carried out, and for each assay triplicate runs were performed.

Determination of the isoelectric point of ASNase. The isoelectric point (IP) of ASNase was determined by the measurements of zeta potential of aqueous solutions of ASNase in a wide $\mathrm{pH}$ range, between 3 and 11 (pH adjusted with $\mathrm{HCl}$ and $\mathrm{NaOH}$ solutions), using a Malvern Zetasizer Nano ZS (Malvern Instruments Ltd. Malvern) instrument at room temperature $\left(25^{\circ} \mathrm{C}\right)$. Aqueous solutions of $\mathrm{NaOH}$ or $\mathrm{HCl} 0.01 \mathrm{M}$ were used to adjust the $\mathrm{pH}$ of the ASNase solutions. 
MWCNTs and ASNase-MWCNTs bioconjugate characterization. The textural properties of the MWCNT materials were determined by $\mathrm{N}_{2}$ adsorption-desorption at $-196{ }^{\circ} \mathrm{C}$ in the relative pressure range 0.05-0.15 on a Quantachrome NOVA 4200e apparatus. Before analysis, each sample (c.a. $100 \mathrm{mg}$ ) was degassed in vacuum at $120^{\circ} \mathrm{C}$ for $3 \mathrm{~h}$. The specific surface area $\left(S_{B E T}\right)$ was calculated by multipoint BET analysis of the obtained data.

Temperature programmed desorption (TPD) analysis was carried out by heating the samples to $1100{ }^{\circ} \mathrm{C}$ at $5^{\circ} \mathrm{C} \mathrm{m^{-1 }}$ under helium flow using an AMI-300 Catalyst Characterization Instrument (Altamira Instruments). The total amounts of $\mathrm{CO}$ and $\mathrm{CO}_{2}$ evolved from the samples were obtained by integration of the TPD spectra. The oxygen content was determined using a rapid oxy cube analyzer (Elementar $\mathrm{GmbH}$ ), in which the sample underwent pyrolysis at $1450^{\circ} \mathrm{C}$. Each sample was analyzed in triplicate. Thermogravimetric (TG) analysis studies were performed on an STA $490 \mathrm{PC} / 4 / \mathrm{H}$ Luxx Netzsch thermal instrument. For each analysis, samples with approximately $10 \mathrm{mg}$ were loaded on an alumina crucible and heated at $10^{\circ} \mathrm{C} \mathrm{min}{ }^{-1}$ from $50{ }^{\circ} \mathrm{C}$ to $900{ }^{\circ} \mathrm{C}$ under airflow, while the weight was measured and recorded continuously.

Raman spectra were recorded in a Brucker RFS100/S FT-Raman spectrometer (Nd:YAG laser, $1064 \mathrm{~nm}$ excitation), at a power of $200 \mathrm{mV}$, with 3000 scans at a resolution of $4 \mathrm{~cm}^{-1}$.

A JEOL 2010F analytical electron microscope, equipped with a field-emission gun, was used for transmission electron microscopy (TEM) images.

Received: 3 August 2021; Accepted: 19 October 2021

Published online: 02 November 2021

\section{References}

1. Castro, D. et al. L-asparaginase production review: Bioprocess design and biochemical characteristics. Appl. Microbiol. Biotechnol. 105, 4515-4534 (2021).

2. Muneer, F. et al. Microbial l-asparaginase: Purification, characterization and applications. Arch. Microbiol. 3, 1-15 (2020).

3. Ulu, A. \& Ates, B. Immobilization of l-Asparaginase on carrier materials: A comprehensive review. Bioconjug. Chem. 28, 1598-1610 (2017).

4. Richards, N. G. J. \& Kilberg, M. S. Asparagine synthetase chemotherapy. Annu. Rev. Biochem. 75, 629-654 (2006).

5. Li, J., Wang, J. \& Bachas, L. G. Biosensor for asparagine using a thermostable recombinant asparaginase from Archaeoglobus fulgidus. Anal. Chem. 74, 3336-3341 (2002).

6. Mohamad, N. R., Marzuki, N. H. C., Buang, N. A., Huyop, F. \& Wahab, R. A. An overview of technologies for immobilization of enzymes and surface analysis techniques for immobilized enzymes. Biotechnol. Biotechnol. Equip. 29, 205-220 (2015).

7. Monajati, M., Borandeh, S., Hesami, A., Mansouri, D. \& Tamaddon, A. M. Immobilization of l-asparaginase on aspartic acid functionalized graphene oxide nanosheet: Enzyme kinetics and stability studies. Chem. Eng. J. 354, 1153-1163 (2018).

8. Venkataraman, A., Amadi, E. V., Chen, Y. \& Papadopoulos, C. Carbon nanotube assembly and integration for applications. Nanoscale Res. Lett. 14, 220 (2019).

9. Balasubramanian, K. \& Burghard, M. Chemically functionalized carbon nanotubes. Small 1, 180-192 (2005).

10. Merum, S., Veluru, J. B. \& Seeram, R. Functionalized carbon nanotubes in bio-world: Applications, limitations and future directions. Mater. Sci. Eng. B 223, 43-63 (2017).

11. Korri-Youssoufi, H., Zribi, B., Miodek, A. \& Haghiri-Gosnet, A.-M. Chapter 4: Carbon-based nanomaterials for electrochemical DNA sensing. In Advanced Nanomaterials (eds Nikolelis, D. P. et al.) 113-150 (Elsevier, 2018).

12. Costa, J. B. et al. Enhanced biocatalytic sustainability of laccase by immobilization on functionalized carbon nanotubes/polysulfone membranes. Chem. Eng. J. 355, 974-985 (2019).

13. Zeinabad, H. A., Zarrabian, A., Saboury, A. A., Alizadeh, A. M. \& Falahati, M. Interaction of single and multi wall carbon nanotubes with the biological systems: Tau protein and PC12 cells as targets. Sci. Rep. 6, 26508 (2016).

14. Zdarta, J., Meyer, A. S., Jesionowski, T. \& Pinelo, M. A general overview of support materials for enzyme immobilization: Characteristics, properties, practical utility. Catalysts 8, 92 (2018).

15. Zdarta, J., Meyer, A. S., Jesionowski, T. \& Pinelo, M. Developments in support materials for immobilization of oxidoreductases: A comprehensive review. Adv. Colloid Interface Sci. 258, 1-20 (2018).

16. Siepi, M. et al. Denatured lysozyme-coated carbon nanotubes: A versatile biohybrid material. Sci. Rep. 9, 16643 (2019).

17. Ebrahim-Habibi, M.-B., Ghobeh, M., Mahyari, F. A., Rafii-Tabar, H. \& Sasanpour, P. An investigation into non-covalent functionalization of a single-walled carbon nanotube and a graphene sheet with protein G: A combined experimental and molecular dynamics study. Sci. Rep. 9, 1273 (2019).

18. Yüce, M., Uysal, E. \& Budak, H. Amplification yield enhancement of short DNA templates using bulk and surface-attached aminefunctionalized single-wall carbon nanotubes. Appl. Surf. Sci. 349, 147-155 (2015).

19. Pedrosa, V. A. et al. Enhanced stability of enzyme organophosphate hydrolase interfaced on the carbon nanotubes. Colloids Surf. B 77, 69-74 (2010).

20. Guan, J. et al. Spectroscopic investigations on the interaction between carbon nanotubes and catalase on molecular level. J. Biochem. Mol. Toxicol. 28, 211-216 (2014).

21. Azevedo, R. M. et al. A strategy for improving peroxidase stability via immobilization on surface modified multi-walled carbon nanotubes. J. Chem. Technol. Biotechnol. 90, 1570-1578 (2015).

22. Silva, C. G. et al. Controlling the surface chemistry of multiwalled carbon nanotubes for the production of highly efficient and stable laccase-based biocatalysts. ChemPlusChem 79, 1116-1122 (2014).

23. Zhuotong, Z. et al. Interaction of tetramer protein with carbon nanotubes. Appl. Surf. Sci. 464, 30-35 (2018).

24. Mottaghitalab, F. et al. The effect of fibronectin on structural and biological properties of single walled carbon nanotube. Appl. Surf. Sci. 339, 85-93 (2015).

25. Wang, Z. et al. Mussel byssus-inspired engineering of synergistic nanointerfacial interactions as sacrificial bonds into carbon nanotube-reinforced soy protein/nanofibrillated cellulose nanocomposites: Versatile mechanical enhancement. Appl. Surf. Sci. 434, 1086-1100 (2018).

26. Saifuddin, N., Raziah, A. Z. \& Junizah, A. R. Carbon nanotubes: A review on structure and their interaction with proteins. J. Chem. 2013, 676815 (2013).

27. Dong, C. et al. Effects of acid treatment on structure, properties and biocompatibility of carbon nanotubes. Appl. Surf. Sci. 264, 261-268 (2013).

28. Jain, S. et al. Toxicity of multiwalled carbon nanotubes with end defects critically depends on their functionalization density. Chem. Res. Toxicol. 24, 2028-2039 (2011). 
29. Cristóvão, R. O. et al. Development and characterization of a novel l-asparaginase/MWCNT nanobioconjugate. RSC Adv. 10, 31205-31213 (2020)

30. Ulu, A. et al. The carboxylated multi-walled carbon nanotubes/l-asparaginase doped calcium-alginate beads: Structural and biocatalytic characterization. Catal. Letters https://doi.org/10.1007/s10562-019-03069-y (2019).

31. Haroun, A., Ahmed, H., Mossa, A.-T., Mohafrash, S. \& Ahmed, E. Production, characterization and immobilization of Aspergillus versicolor L-asparaginase onto multi-walled carbon nanotubes. Biointerface Res. Appl. Chem. 10, 5733-5740 (2020).

32. Senthil Kumar, M. \& Selvam, K. Isolation and Purification of high efficiency L-asparaginase by quantitative preparative continuouselution SDS PAGE electrophoresis. J. Microb. Biochem. Technol. 3, 73-83 (2011).

33. Feng, W. \& Ji, P. Enzymes immobilized on carbon nanotubes. Biotechnol. Adv. 29, 889-895 (2011).

34. Ashrafi, H. et al. Nanostructure l-asparaginase-fatty acid bioconjugate: Synthesis, preformulation study and biological assessment. Int. J. Biol. Macromol. 62, 180-187 (2013).

35. Tabandeh, M. R. \& Aminlari, M. Synthesis, physicochemical and immunological properties of oxidized inulin-l-asparaginase bioconjugate. J. Biotechnol. 141, 189-195 (2009).

36. Dinu, C. Z. et al. Enzyme-based nanoscale composites for use as active decontamination surfaces. Adv. Funct. Mater. 20, 392-398 (2010).

37. El-Naggar, N.E.-A., Deraz, S. F., El-Ewasy, S. M. \& Suddek, G. M. Purification, characterization and immunogenicity assessment of glutaminase free L-asparaginase from Streptomyces brollosae NEAE-115. BMC Pharmacol. Toxicol. 19, 51 (2018).

38. Noma, S. A. A., Ulu, A., Koytepe, S. \& Ateș, B. Preparation and characterization of amino and carboxyl functionalized core-shell Fe3O4/SiO2 for L-asparaginase immobilization: A comparison study. Biocatal. Biotransform. 38, 392-404 (2020).

39. Orhan, H. \& Aktaş Uygun, D. Immobilization of L-asparaginase on magnetic nanoparticles for cancer treatment. Appl. Biochem. Biotechnol. 191, 1432-1443 (2020).

40. Monthioux, M. et al. Sensitivity of single-wall carbon nanotubes to chemical processing: An electron microscopy investigation. Carbon N. Y. 39, 1251-1272 (2001).

41. Figueiredo, J. L. \& Pereira, M. F. R. The role of surface chemistry in catalysis with carbons. Catal. Today 150, 2-7 (2010).

42. Figueiredo, J. L., Pereira, M. F. R., Freitas, M. M. A. \& Órfão, J. J. M. Modification of the surface chemistry of activated carbons. Carbon N. Y. 37, 1379-1389 (1999).

43. Figueiredo, J. L., Pereira, M. F. R., Freitas, M. M. A. \& Órfão, J. J. M. Characterization of active sites on carbon catalysts. Ind. Eng. Chem. Res. 46, 4110-4115 (2007).

44. Shakambari, G. et al. Cloning and expression of L-asparaginase from Bacillus tequilensis PV9W and therapeutic efficacy of Solid Lipid Particle formulations against cancer. Sci. Rep. 8, 18013 (2018).

45. Vasudev, S. S. et al. Formulation of PEG-ylated L-asparaginase loaded poly (lactide-co-glycolide) nanoparticles: Influence of Pegylation on enzyme loading, activity and in vitro release. Pharmazie 66, 956-960 (2011).

46. Iyer, P. V. \& Ananthanarayan, L. Enzyme stability and stabilization: Aqueous and non-aqueous environment. Process Biochem. 43, 1019-1032 (2008).

47. Likodimos, V. et al. Controlled surface functionalization of multiwall carbon nanotubes by HNO3 hydrothermal oxidation. Carbon N. Y. 69, 311-326 (2014)

48. Magri, A. et al. A critical analysis of L-asparaginase activity quantification methods-colorimetric methods versus high-performance liquid chromatography. Anal. Bioanal. Chem. 410, 6985-6990 (2018).

49. Belhachemi, M. \& Addoun, F. Comparative adsorption isotherms and modeling of methylene blue onto activated carbons. Appl. Water Sci. 1, 111-117 (2011).

\section{Acknowledgements}

This work was developed within the scope of the project CICECO-Aveiro Institute of Materials, UIDB/50011/2020 \& UIDP/50011/2020, financed by national funds through the Portuguese Foundation for Science and Technology/MCTES. This work was also financially supported by Base-UIDB/50020/2020 and ProgrammaticUIDP/50020/2020 Funding of LSRE-LCM, funded by national funds through FCT/MCTES (PIDDAC), and POCI-01-0145-FEDER-031268-funded by FEDER, through COMPETE2020-Programa Operacional Competitividade e Internacionalização (POCI), and by national funds (OE), through FCT/MCTES. Márcia C. Neves acknowledges the research contract CEECIND/00383/2017. Valéria C. Santos-Ebinuma acknowledges FAPESP (2018/06908-8). Ana P. M. Tavares acknowledges the FCT for the research contract CEECIND/2020/01867. Raquel O. Cristóvão acknowledges FCT funding under DL57/2016 Transitory Norm Programme. Maria A. Barros acknowledges the research grant from FCT (Ref. SFRH/BD/145014/2019). João C. F. Nunes acknowledges SPQ and FCT for the PhD fellowship (SFRH/BD/150671/2020).

\section{Author contributions}

M.R.A., R.O.C., M.A.B., J.C.F.N., and M.C.N.: Methodology, Investigation. M.R.A. and R.O.C.: Writing-original draft. R.O.C, R.A.R.B., J.M.L., J.L.F., M.G.F., V.C.S.E., A.P.M.T., and C.G.S.: Methodology, Investigation, Writing-review and editing. A.P.M.T. and C.G.S: Supervision.

\section{Competing interests}

The authors declare no competing interests.

\section{Additional information}

Supplementary Information The online version contains supplementary material available at https://doi.org/ 10.1038/s41598-021-00841-2.

Correspondence and requests for materials should be addressed to A.P.M.T. or C.G.S.

Reprints and permissions information is available at www.nature.com/reprints.

Publisher's note Springer Nature remains neutral with regard to jurisdictional claims in published maps and institutional affiliations. 
(c) (i) Open Access This article is licensed under a Creative Commons Attribution 4.0 International cc) License, which permits use, sharing, adaptation, distribution and reproduction in any medium or format, as long as you give appropriate credit to the original author(s) and the source, provide a link to the Creative Commons licence, and indicate if changes were made. The images or other third party material in this article are included in the article's Creative Commons licence, unless indicated otherwise in a credit line to the material. If material is not included in the article's Creative Commons licence and your intended use is not permitted by statutory regulation or exceeds the permitted use, you will need to obtain permission directly from the copyright holder. To view a copy of this licence, visit http://creativecommons.org/licenses/by/4.0/.

(C) The Author(s) 2021 